\title{
Life Cycle Assessment of Polyurethane Foams from Polyols Obtained through Chemical Recycling
}

\author{
Alessandro Marson, Massimiliano Masiero, Michele Modesti, Antonio Scipioni, \\ and Alessandro Manzardo*
}

Cite This: ACS Omega 2021, 6, 1718-1724

Read Online

ABSTRACT: In this research, the results of the life cycle assessment of polyurethane (PUR) foams with different recycled polyol contents are presented. A methodological framework implementing laboratory activities directly into the life cycle assessment has been developed. Laboratory activities made the primary data related to the recycled polyol production available through the glycolysis of polyurethane scraps and the subsequent production and characterization of the foams. Five different formulations were analyzed with glycolyzed polyol content ranging from 0 to $100 \%$. A comprehensive set of impact categories was considered. To ensure the robustness of the results, the influence of two different end-of-life allocation approaches was investigated, and the model was subjected to sensitivity and uncertainty

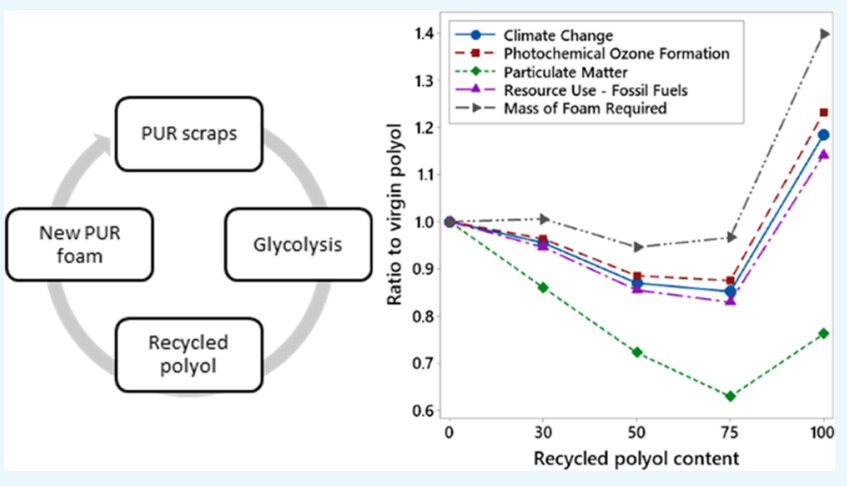
analyses. Formulations with recycled content of 50 and $75 \%$ scored better environmental impacts compared to others. The main contributions to the overall impact resulted to be related to the production of isocyanate and virgin polyol. Physical characteristics such as density and thermal conductivity emerged as the main variables to be considered to minimize the overall environmental impacts of PUR foams.

\section{INTRODUCTION}

Plastics are fundamental in several production systems; however, at the same time, they are recognized as a source of severe environmental burden. ${ }^{1,2}$ Despite this, global production volumes have been growing steadily since 1950, reaching a total of almost 360 million tons in $2018 .^{3}$ On the same line, the interest of institutions, scientific research, ${ }^{1}$ and general public has increased. ${ }^{4}$ The main environmental critical issues related to plastics are their low recycling rate, high landfilled fraction, use of hazardous additives, and potential dispersion of macro- and microplastics into the environment (e.g., water bodies). ${ }^{5-7}$

To tackle these issues, the European Commission in 2015 presented the circular economy action plan, ${ }^{5}$ followed in 2018 by a specific plastic's strategy. ${ }^{8}$ A public consultation has identified, among others, two key messages: the importance of providing information relating to the potential environmental impacts of plastics and the need to tackle their potential environmental impacts already in the early stage of their life cycle, such as in the design and development phase. ${ }^{9}$ In the circular economy action plan, particular attention has been paid to the building and construction sector, contemplating different possibilities to achieve sustainable plastic use and production using biobased and/or recycled feedstock. However, the commission also recognized that the potential environmental benefits of such solutions should be further investigated. To do so, the adoption of the life cycle assessment (LCA) method is recommended. The LCA has proved to be effective in supporting informed decisions for the improvement of the environmental performance of products and services ${ }^{10}$ and has already been applied ${ }^{11,12}$ to evaluate the potential environmental impacts related to polymer production. Furthermore, the tool has proven to be effective in the design and development of new building materials. ${ }^{13,14}$

Among the different plastics, rigid polyurethane (PUR) foam for thermal insulation, due to its application and market share, ${ }^{3}$ is at the center of research for the development of new biobased and/or recycled formula.

The production of partially biobased PUR foams suitable for thermal insulation has been widely discussed in the literature (e.g., using polyols derived from renewable resources as cashew nut ${ }^{15}$ and castor oil). ${ }^{16}$ However, the number of studies on their environmental performances is still limited. Manzardo et

Received: December 1, 2020

Accepted: December 25, 2020

Published: January 7, 2021 
al. investigated the environmental performance of PUR containing polyol derived from azelaic acid and lignin, proving that biobased formulations can have more significant impacts than their fossil counterpart. ${ }^{14}$ In the same study, Manzardo et al. ${ }^{14}$ proposed a methodological framework for implementing an early-stage assessment during laboratory activities. Fridrihsone et al. presented a cradle-to-gate study on vegetable polyols (rapeseed oil) intended for the production of PUR foams. ${ }^{17}$ In this case, biobased formulations resulted in environmental hotspots with reference to water quality impact assessment categories. In the same study, Fridrihsone et al. highlighted that the choice of the allocation approach in biobased feedstock production can significantly influence the impact assessment results. ${ }^{17}$

The foams with recycled content can be produced using polyols obtained from PUR's chemical recycling. ${ }^{18}$ In particular, among different techniques, glycolysis is the most widely used for PU. ${ }^{19}$ Polyol recovered through glycolysis can be used at different substitution levels, depending on the application: low-density rigid noninsulation foam can handle over $50 \%$ of the recycled polyol. ${ }^{20}$ Other applications like reinforced structural rigid foam or high-density structural PUR can have only $25 \%$ of the recycled polyol without substantial changes compared to the standard. ${ }^{20}$ Despite these promising results, no studies have been published on the potential environmental impacts of PUR containing polyols recycled from glycolysis. One of the main reasons is that, considering that the research on these materials is ongoing, there is no framework to support an LCA assessment in their design and development phase. In this context, considering the potential market development of recycled PUR foams in the future, ${ }^{21}$ there is a need to develop an LCA framework to assess their potential environmental impacts.

Therefore, the objective of the research can be formulated as follows:

- Develop an LCA methodological framework to assess the potential environmental impacts of PUR produced from chemically recycled polyols in the early stage of the design and development of new formulations.

- Verify and discuss its application to evaluate the environmental performance of PUR foams containing recycled polyol from glycolysis.

\section{RESULTS AND DISCUSSION}

Foam Characterization. Foam density and conductivity are reported in Table 1 . All of the foams have a density between 37 and $40 \mathrm{~kg} / \mathrm{m}^{3}$ and good dimensional stability. The foam with recycled polyol between 30 and $75 \%$ has a thermal conductivity value like the reference one (R0). Nevertheless, increasing the amount of recycled polyol leads to a worse cellular structure that results in a higher thermal conductivity

Table 1. Physical Properties of the Foam and Mass Required to Satisfy the Functional Unit

\begin{tabular}{lccc} 
foam & $\begin{array}{c}\text { density } \\
{\left[\mathrm{kg} / \mathrm{m}^{3}\right]}\end{array}$ & $\begin{array}{c}\text { thermal conductivity } \\
{[\mathrm{W} / \mathrm{mK}]}\end{array}$ & $\begin{array}{c}\text { mass required } \\
{[\mathrm{kg}]}\end{array}$ \\
\hline R0 & $3.89 \times 10^{1}$ & $2.28 \times 10^{-2}$ & $8.87 \times 10^{-1}$ \\
R30 & $3.93 \times 10^{1}$ & $2.27 \times 10^{-2}$ & $8.92 \times 10^{-1}$ \\
R50 & $3.73 \times 10^{1}$ & $2.25 \times 10^{-2}$ & $8.39 \times 10^{-1}$ \\
R75 & $3.76 \times 10^{1}$ & $2.28 \times 10^{-2}$ & $8.57 \times 10^{-1}$ \\
R100 & $3.88 \times 10^{1}$ & $3.19 \times 10^{-2}$ & $1.24 \times 10^{0}$
\end{tabular}

at $100 \%$ substitution (scanning electron microscopy (SEM) analysis of the foams are reported from Figures S1-S5). Furthermore, the increase in the glycolyzed polyol leads to an increase in the ratio between methylene diphenyl diisocyanate (MDI) and polyol.

Life Cycle Impact Assessment Results. Impact assessment is reported in Table 2. Figure 1 presents the relative results with respect to the reference formulation R0 for the considered allocation approaches. The results of the entire set of impact categories are reported in Table S1.

The introduction of a recycled polyol resulted in an overall reduction in the environmental impact up to the replacement rate of $75 \%$. Above this rate, there is a significant drop in the physical characteristics of the foam and a consequent worsening of the environmental performance. R75 and R50 resulted in the formulation with better overall environmental performance. The use of different allocation approaches did not significantly influence the impact assessment results.

Regarding the main impact assessment categories investigated, compared to R0, R75 was the best formulation with reference to CC $(-15 \%)$, POF $(-12 \%)$, RUF $(-17 \%)$, and PM (-37\%).

Regarding the other impact assessment categories investigated (see the Supporting Information), compared to R0, R75 resulted to be the best formulation with reference to human health effects $(-20 \%)$, acidification terrestrial and freshwater $(-12 \%)$, eutrophication marine $(-12 \%)$, eutrophication terrestrial $(-12 \%)$, ecotoxicity freshwater $(-16 \%)$, water scarcity $(-29 \%)$, climate change-fossil $(-15 \%)$, and climate change-biogenic (-47\%). In the remaining categories, compared to R0, R50 was the best formulation with reference to ozone depletion $(-4 \%)$, noncancer human health effects $(-8 \%)$, eutrophication freshwater $(-6 \%)$, land use $(-2 \%)$, resource use - mineral and metals $(-2 \%)$, and climate change-land use and transformation $(-1 \%)$.

R0 formulation scored better performance only for the category ionizing radiation.

Interpretation. The results of the contribution analysis of the formulation R0 and R100 are represented in Figure S6 for the cutoff allocation approaches, while results for 50:50 allocation approaches are reported in Figure S7.

Considering the $\mathrm{R} 0$ formulation, the MDI production resulted to be the main source of impacts for CC (58\%), POF (68\%), and RUF (66\%), while virgin polyol had the greatest contribution (50\%) to PM. This fact justifies the significant impact reduction scored by R75 compared to R0 in the PM category. Foam production and end-of-life stages contributed less than $20 \%$. The introduction of a recycled material involves a gradual reduction of the virgin polyol's contribution and, consequently, an increase in other reagents/ phases, including glycolyzed polyol. For R100, MDI had the largest contribution in all impact categories, while glycolyzed polyol had a smaller contribution (between 5 and 9\%).

The results of sensitivity analysis proved that physical characteristics (density and thermal conductivity) are the main variables to influence the impact assessment indicator results, while parameters describing foam formulation and recycled content have limited relevance (Table S2). These results are in line with those described by Manzardo et al. ${ }^{14}$ in the case of biobased PUR.

The results of uncertainty analysis confirmed that R50 and R75 have lower environmental impacts than R0 in 18 and 14 impact categories, respectively, out of 19 considering the 50:50 
$8 \stackrel{\circ}{\circ}$

$8 \times \vec{x} \times \vec{x}$

ஸิ $\infty \circ$

$\therefore$ ㅇํㅇ

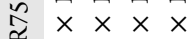

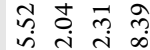

$\therefore$ iิ

\) $\times \vec{x} \times \vec{x}$

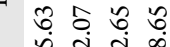

$\therefore$ 1ิ

$\sqrt{\circ} \times \times \times$

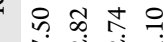

$\therefore$ iิ

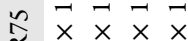

?ำ

$\therefore$ ํํ응

$\stackrel{x}{x} \times \times \times$

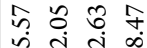

$\therefore$ 1 00

이사 $\times$

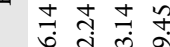

$\therefore$ ํํ웅

\&ิ $\times \times \times \times$

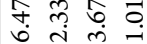

节

강

$\checkmark \mathrm{O}$

茫

○ $\sum_{Z}$

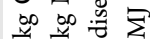

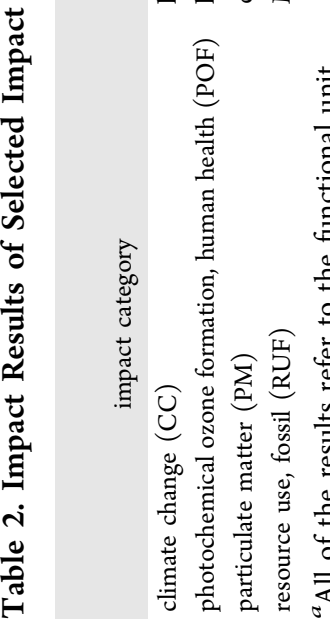

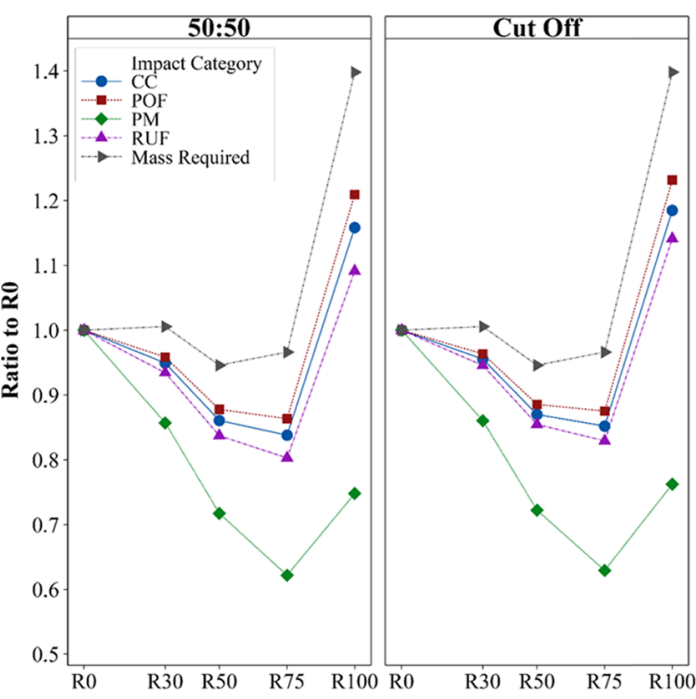

Figure 1. Relative results with respect to the reference foam R0. All of the results refer to the functional unit.

allocation approach, while 17 and 13 considering the cutoff approach, respectively. The comparison between R50 and R75 revealed that the two formulations should be considered equivalent in eight impact categories: cancer human health effects (only for the cutoff approach), ecotoxicity freshwater, eutrophication freshwater, eutrophication marine (only for the cutoff approach), eutrophication terrestrial, noncancer human health effects, POF, and water scarcity.

The complete results of this analysis are reported in Table S3.

Discussion on the Methodological Framework. The results of the study proved the applicability of the proposed methodological framework. The key points of the original model proposed by Manzardo et al. ${ }^{14}$ allowed the identification of the optimum in correspondence with a partial replacement of the virgin polyol with the recycled one. In this regard, Figure S8 shows the results that would have been obtained by merely basing the comparison on the foam mass without considering the thermal insulation properties. It can be noted that, for the same mass, the formulation with $100 \%$ recycled polyol would be characterized by the lowest environmental impacts. This result is in clear contrast to the poor physical characteristics of the formulation.

Considering the modifications introduced to the original framework, the adoption of a wider set of impact categories allowed us to demonstrate that the glycolyzed material does not involve burden-shifting or significant hotspots. However, this integration remains valid, as it will enable alignment with the most recent standards applicable to the analyzed product. $^{22,23}$ This is due to the limited contribution to the total impact of the glycolyzed polyol compared to the other reagents, which never exceeds $9 \%$ (cutoff approach). It emerges that the choice of the allocation approach becomes more relevant as the recycled content (and its contribution on the total impact) increases, as demonstrated by van der Harst et al. $^{24}$ The adoption of different allocation approaches, therefore, should be considered also when biobased materials are implemented as demonstrated by Fridrishone et al. ${ }^{17}$ 


\section{CONCLUSIONS}

This study presents a methodological LCA framework for the assessment of the potential environmental impacts of chemically recycled material and its application in the case of PUR containing glycolyzed polyol. The LCA framework was developed starting from the approach proposed by Manzardo et al. ${ }^{14}$ by introducing two modifications related to the set of impact categories to be investigated and the use of different allocation approaches.

The applicability of the framework was confirmed in the development and testing of five foams with different contents of recycled polyol ranging from 0 to $100 \%$ in the mass of recycled polyol from glycolysis.

The results proved that formulations with 50\% (R50) and $75 \%$ (R75) of recycled polyol content in mass have better environmental performances in most of the environmental impact categories compared to the others (R0, R30, and R100). These two formulations however have similar environmental performances in 13-17 impact categories out of the 19 investigated (depending on the allocation approach). It is therefore not possible to claim the overall environmental superiority of one formula over the other. The uncertainty analysis confirmed these results. Sensitivity analysis proved that impact assessment results strongly depend on the density and thermal conductivity of the PUR foam. It can be concluded that the use of recycled polyol from glycolysis can contribute to the reduction of the potential environmental impacts of PUR foams if compared to the use of virgin polyol, provided that physical and thermal characteristics are guaranteed. This result confirms the outcomes of Manzardo et al. ${ }^{14}$ with reference to biobased PUR foams.

The results of the study also proved that higher recycled content does not necessarily guarantee better environmental performances.

From a methodological point of view, the introduction of the new set of environmental categories did not highlight any burden shift. However, it is recommended to adopt this modification to be in line with the recent normative development. $^{22}$ On the other hand, the use of different allocation approaches should be recommended for recycled content considering that in some cases it might affect the impact assessment results. $^{24}$

\section{METHODOLOGY}

Methodological Framework. LCA is defined as the compilation and evaluation of the inputs, outputs, and potential environmental impacts of a product system throughout its life cycle. ${ }^{25}$ The methodology is structured into four iterative phases: goal and scope definition, inventory analysis, impact assessment, and interpretation of results. In this study, the framework proposed by Manzardo et al. ${ }^{14}$ that integrates the LCA methodology for an assessment of potential environmental impacts of biobased PUR in the design and development phase is used as the ground for the development of the proposal related to the recycled PUR (Figure 2). The key points of the original model proposed by Manzardo et al., ${ }^{14}$ namely, the integration of laboratory activities directly into the LCA (in particular, the design of the experiment implemented in goal and scope definition) and the choice of a functional unit that takes into account the physical performances of the insulating material are considered valid in the

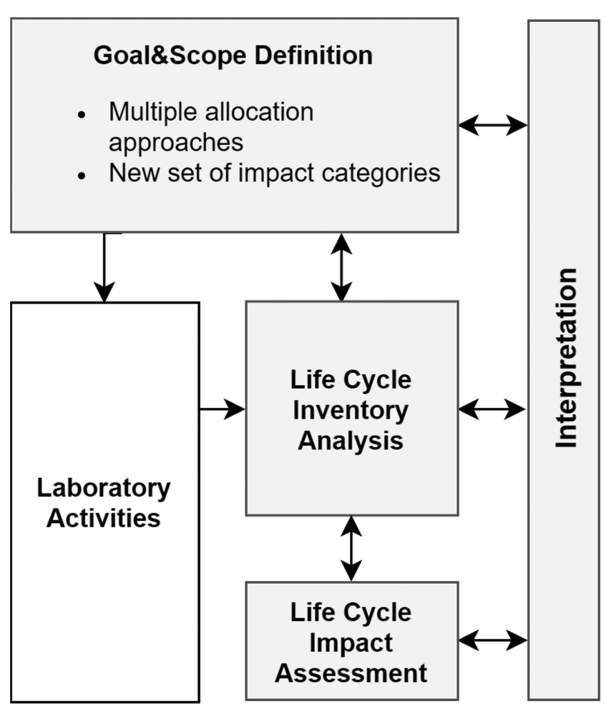

Figure 2. Framework proposal for the evaluation of recycled PUR in the early stage of design and development.

methodological proposal of this research, while the following modifications are introduced (Figure 2):

- The set of impact assessment categories is substituted according to the recent EN 15804 standard for the assessment of the environmental sustainability of

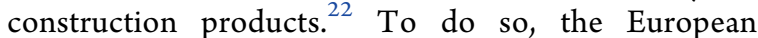
Footprint method $2.0^{26}$ was considered.

- Different allocation approaches are used in parallel. This solution is adopted to verify the influence of the recycled feedstocks' modeling on the life cycle impact assessment results. Research from van der Harst et al. ${ }^{24}$ proved that the recycled content impact assessment results are highly influenced by the selection of allocation approaches. Moreover, according to Allacker et al., more than 11 allocation approaches can be identified in the LCA. ${ }^{27}$

Goal and Scope. The goal of the study is to perform an assessment of the potential environmental impacts of PUR formulations with different recycled contents by applying the proposed methodological framework. The intended use of the PUR understudy is the thermal insulation in buildings. Consequently, EN $15804^{22}$ and EN $16783,^{28}$ as well as the "Product Environmental Footprint Category Rules (PEFCRs) for thermal insulation", were considered in this study. ${ }^{23}$

The declared unit is defined as the amount of foam needed to achieve $1 \mathrm{~m}^{2} \mathrm{~K} / \mathrm{W}$ of thermal resistance $R$, defined as

$$
R=L / \lambda
$$

where $\lambda$ is the thermal conductivity $[\mathrm{W} /(\mathrm{K} \cdot \mathrm{m})]$ and $L$ is the thickness of the insulation layer $[\mathrm{m}]$. The behavior of physical performance over time is assumed to be homogeneous for all foams. Therefore, considering the comparative nature of the study, this variable is not considered.

The system boundaries include the processes of extraction, production, transport of raw materials, and foam production process and its management at the end of life. The installation, use, and deconstruction phases were excluded from the study according to EN $16783^{28}$ (Figure 3).

The main raw materials used in the manufacturing of the PUR are virgin polyol(s), recycled polyol(s), and isocyanate. These materials are mixed with a catalyst, foaming agents, and surfactants to produce the PUR foams. 


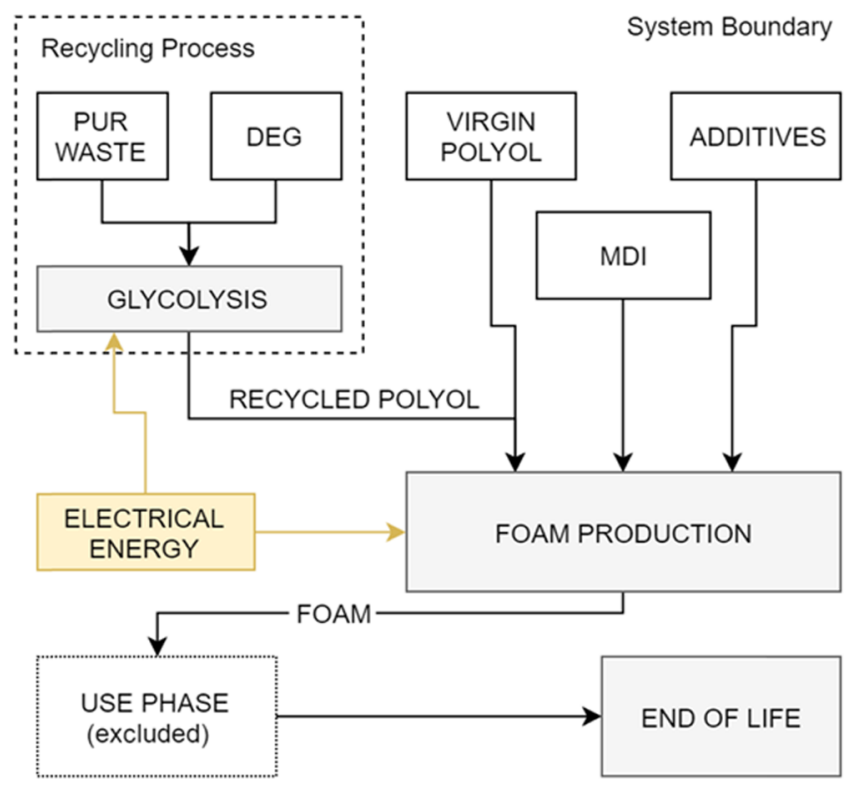

Figure 3. System boundary of the production process.

The recycled polyol derives from the glycolysis of industrial PUR scraps, diethylene glycol (DEG), and catalyst. These materials are mixed at high temperatures and react to form a single-phase polyol with a high hydroxyl number.

To have a complete view of the environmental impact of the effects of the introduction of the recycled polyol in PUR foams, different replacement levels of the virgin polyol are considered $(0,30,50,75$, and $100 \%)$. All of the analyzed formulations were developed at a laboratory scale to guarantee the same conditions. Laboratory activities were in fact performed to acquire primary data on the raw material consumption, the energy consumption, the emissions, and the physical characteristics (such as density and conductivity) of the final PUR foams. Secondary data related to raw material production and end-of-life processes were derived from Ecoinvent $3.5^{29}$ database. The model was developed using SimaPro ${ }^{30}$ version 9.0 .

To facilitate the interpretation of results, in this paper, only information related to the most relevant impact categories according to the reference PEFCR ${ }^{23}$ (Table 3 ) is presented in the following sections. The complete list of impact assessment categories is reported in Table S4.

With reference to allocation, two approaches are selected in this study: the so-called cutoff approach, due to its diffusion in environmental product declaration of construction products, and the 50:50 approach, according to SETAC recommendation. ${ }^{27}$ In the cutoff approach, all of the burdens derived from the recycling process are allocated to the system using the

Table 3. Impact Categories and Related Methods Reported in This Paper

\begin{tabular}{|c|c|c|}
\hline impact category & unit & method \\
\hline climate change (CC) & $\mathrm{kg} \mathrm{CO} \mathrm{CO}_{2}$ eq & $\begin{array}{l}\text { baseline model of } 100 \\
\text { years of the IPCC }\end{array}$ \\
\hline particulate matter (PM) & disease incidence & $\begin{array}{l}\text { UNEP-recommended } \\
\text { model }^{31}\end{array}$ \\
\hline $\begin{array}{l}\text { photochemical ozone } \\
\text { formation, human health } \\
\text { (POF) }\end{array}$ & $\mathrm{kg}$ NMVOC eq & $\begin{array}{l}\text { LOTOS-EUROS } \\
\text { model }^{32}\end{array}$ \\
\hline resource use, fossil (RUF) & MJ & CML $2002^{33}$ \\
\hline
\end{tabular}

recycled material. In the 50:50 approach, the impacts are divided equally between the system that produces the waste and the system that uses the recycled material.

Equations describing the two approaches are reported in eq 2 (cutoff) and eq 3 (50:50)

$$
\begin{aligned}
& \left(1-R_{1}\right) \cdot E_{\mathrm{V}}+R_{1} \cdot E_{\text {recycled }} \\
& \left(1-R_{1}\right) \cdot E_{\mathrm{V}}+\frac{R_{1}}{2} \cdot E_{\text {recycled }}
\end{aligned}
$$

where $R_{1}$ is the recycled content, $E_{\mathrm{V}}$ is the specific emissions and resources consumed (per functional unit) arising from the acquisition and preprocessing of virgin material, and $E_{\text {recycled }}$ is the specific emissions and resources consumed (per functional unit) arising from the recycling process, including collection, sorting, and transportation processes. ${ }^{26}$

Inventory Analysis. Laboratory Activities and Material Characterization. PU foams are obtained starting from Alcupol $3810(n \mathrm{OH}=380 \mathrm{mg} \mathrm{KOH} / \mathrm{g})$, Isoexter 4530 $(n \mathrm{OH}=510 \mathrm{mg} \mathrm{KOH} / \mathrm{g})$ (both by COIM, Italy), and glycolyzed polyol. The last one is produced from diethylene glycol (DEG) (COIM, Italy) mixed at $200{ }^{\circ} \mathrm{C}$ with PUR scraps (ratio between the masses of PUR scraps and DEG is 1.5) and a proper selective catalyst ( $2 \%$ by weight). These materials are mixed for $4 \mathrm{~h}$ and react to form a single-phase polyol with a high hydroxyl number $(n \mathrm{OH})$, measured with a potentiometric method and result in $522 \mathrm{mg} \mathrm{KOH} / \mathrm{g}$, with a viscosity of about $3000 \mathrm{cP}$. The product obtained is a kind of mixture of different types of polyols, glycolysis agents, and isocyanates derivatives. ${ }^{19}$ In this research, only one type of PUR scraps has been used. The electricity consumption for the entire glycolysis process was equal to $0.167 \mathrm{kWh} / \mathrm{kg}$ of polyol obtained.

Hydroxyl number is obtained through potentiometric analysis. Free aromatic amines could arise by hydrolysis reactions and their content must be lower than $0.1 \%$. This is verified through high-performance liquid chromatography (HPLC) analysis.

Before the reaction with isocyanate, the following reagents are added to the polyol: foaming agent (water and HFO), surfactants (TEGOSTAB B8485 and Dabco EM400 both by Evonik Industries, Germany), and catalyst ( $\mathrm{N}, \mathrm{N}$-dimethylcyclohexylamine (DMCHA) and Dabco BL-11 both by Air Product Inc.).

After homogenization, isocyanate (Isocom M, by COIM, Italy) is added, vigorously mixed, and then poured in a preheated open mold (preheated at $40{ }^{\circ} \mathrm{C}$ ). In less than a minute, the product is formed that is dimensionally stable, and it is kept at $70{ }^{\circ} \mathrm{C}$ for $8 \mathrm{~h}$. Data related to the foam nomenclature and formulation are reported in Table 4. In particular, the number reported in the name of the foam represents the percentage of the recycled polyol content.

Apparent density and thermal conductivity were measured according to ISO 845 and ISO 8301, respectively.

Life Cycle Inventory Analysis and Modeling. The quantity of PUR needed to satisfy the functional unit was calculated for each foam based on the density and conductivity resulting from laboratory activities. The required amount of foam $M_{\mathrm{PU}}$ was derived using

$$
M_{\mathrm{PU}}=\rho \cdot A \cdot \lambda \cdot R
$$

where $A$ is the surface to be covered with an insulating material, equal to $1 \mathrm{~m}^{2}$. The upstream processes, such as the production of polyols, isocyanate, DEG, and all of the 
Table 4. Quantity of the Reagents to Produce $1 \mathrm{~kg}$ of PUR Foams

\begin{tabular}{|c|c|c|c|c|c|c|}
\hline \multirow[b]{2}{*}{ reagent } & \multirow[b]{2}{*}{ unit } & \multicolumn{5}{|c|}{ foam } \\
\hline & & R0 & $\mathrm{R} 30$ & R50 & R75 & $\mathrm{R} 100$ \\
\hline polyol (Alcupol 3810) & $\mathrm{kg}$ & $1.95 \times 10^{-1}$ & $1.34 \times 10^{-1}$ & $9.39 \times 10^{-2}$ & $4.59 \times 10^{-2}$ & \\
\hline polyol (Isoexter 4530) & $\mathrm{kg}$ & $1.95 \times 10^{-1}$ & $1.34 \times 10^{-1}$ & $9.39 \times 10^{-2}$ & $4.59 \times 10^{-2}$ & \\
\hline glycolyzed polyol & $\mathrm{kg}$ & & $1.14 \times 10^{-1}$ & $1.88 \times 10^{-1}$ & $2.77 \times 10^{-1}$ & $3.62 \times 10^{-1}$ \\
\hline catalyst (BL-11) & $\mathrm{kg}$ & $1.17 \times 10^{-3}$ & & & & \\
\hline catalyst (DMCHA) & $\mathrm{kg}$ & $5.86 \times 10^{-3}$ & $3.49 \times 10^{-3}$ & $2.47 \times 10^{-3}$ & $2.42 \times 10^{-3}$ & $1.67 \times 10^{-3}$ \\
\hline surfactants (EM400) & $\mathrm{kg}$ & $3.52 \times 10^{-3}$ & $3.49 \times 10^{-3}$ & $3.71 \times 10^{-3}$ & $3.62 \times 10^{-3}$ & $3.34 \times 10^{-3}$ \\
\hline surfactants (B8485) & $\mathrm{kg}$ & $9.38 \times 10^{-3}$ & $1.16 \times 10^{-2}$ & $1.11 \times 10^{-2}$ & $1.09 \times 10^{-2}$ & $1.09 \times 10^{-2}$ \\
\hline foaming agent (HFO) & $\mathrm{kg}$ & $8.21 \times 10^{-3}$ & $8.14 \times 10^{-3}$ & $7.42 \times 10^{-3}$ & $7.25 \times 10^{-3}$ & $7.52 \times 10^{-3}$ \\
\hline foaming agent (water) & $\mathrm{kg}$ & $3.17 \times 10^{-2}$ & $2.79 \times 10^{-2}$ & $2.97 \times 10^{-2}$ & $2.78 \times 10^{-2}$ & $2.92 \times 10^{-2}$ \\
\hline isocyanate (Isocom M) & $\mathrm{kg}$ & $5.91 \times 10^{-1}$ & $6.00 \times 10^{-1}$ & $6.07 \times 10^{-1}$ & $6.15 \times 10^{-1}$ & $6.22 \times 10^{-1}$ \\
\hline mass of foam & $\mathrm{kg}$ & $1.00 \times 10^{0}$ & $1.00 \times 10^{0}$ & $1.00 \times 10^{0}$ & $1.00 \times 10^{0}$ & $1.00 \times 10^{0}$ \\
\hline
\end{tabular}

additives, are derived from Ecoinvent $\mathrm{v} 3.5^{29}$ database considering the European scenario. For the PUR scraps, only the transport process is considered, following the primary information obtained from the manufacturer as the material is not processed before being used in the glycolysis process. The European energy mix is adopted in the modeling of electricity consumption.

The chemical recycling process is modeled considering the quantity of reagents and energy consumption measured in the laboratory, attributing impacts to the recycled material consistently with the chosen end-of-life allocation approach.

The foam production process is characterized starting from the Ecoinvent v3.5 $5^{29}$ database modified based on the quantity of polyols and isocyanate, as well as additives and emission in air assessed considering that all foaming agents are emitted into the atmosphere. According to the data set, $0.2 \%$ by weight of the foam produced is considered waste.

The transport of raw materials and waste is modeled considering $100 \mathrm{~km}$ and a Euro 3 lorry (16-32 metric ton). The end-of-life scenario is defined according to PEFCR ${ }^{23}$ requirements for "non-mineral insulating materials": $45 \%$ incineration and 55\% landfill. The packaging materials and the installation and dismantling processes are excluded from the analysis due to limited contributions in terms of mass and energy.

Interpretation Approach for the Discussion of Results. Interpretation of the results includes contribution, sensitivity, and uncertainty analyses.

Contribution analysis is performed to identify the main sources of impact along the life cycle of the PUR foams. The following stages were defined:

(1) Glycolyzed polyol: includes the production and procurement of PUR waste and other reagents, as well as the glycolysis process itself.

(2) Virgin polyol: includes the production of the virgin polyol and the transportation to the foam production site.

(3) MDI: includes the production of the isocyanate and the transportation to the foam production site.

(4) Foam production: includes energy consumption and emissions, as well as production and procurement of additives.

(5) End of life: includes all of the processes from PUR dismantling to the final disposal.

Sensitivity analysis is performed to identify the most relevant parameters in the model. Influence of percentage of recycled polyol, $\mathrm{MDI} /$ polyol ratio, and mass required to satisfy the functional unit were investigated by applying an arbitrary variation of $\pm 20 \%$ at the starting parameter (R50 formulation and cutoff allocation approach were considered in such a way as to intercept the effects of both virgin and recycled polyol). Sensitivity $S$ is defined as 5

$$
S=\frac{\Delta I_{i}}{I_{0, i}} / \frac{\Delta p_{j}}{p_{0, j}}
$$

where $\frac{\Delta p_{j}}{p_{0, i}}$ is the considered variation of parameter $j$ (assumed $\pm 20 \%)$ and $\frac{\Delta I_{i}}{I_{0, i}}$ is the normalized variation of the results for the impact category j. A higher sensitivity implies a greater influence of the parameter on the impact category.

Uncertainty analysis is performed to test the solidity of the results. According to a common practice in LCA, the pedigree matrix $^{34}$ approach was adopted, thus conducting a Monte Carlo analysis. Using the functionalities of SimaPro software, pairwise uncertainty analyses were performed (all combinations of R0, R50, and R75), showing in how many calculations a product scored lower than another in a certain impact category. $^{34}$

\section{ASSOCIATED CONTENT}

\section{Supporting Information}

The Supporting Information is available free of charge at https://pubs.acs.org/doi/10.1021/acsomega.0c05844.

SEM analysis; results of life cycle impact assessment; results of sensitivity analysis; results of uncertainty analysis; graphic results of LCA conducted referring to 1 $\mathrm{kg}$ of foam; and the list of impact categories analyzed (PDF)

\section{AUTHOR INFORMATION}

\section{Corresponding Author}

Alessandro Manzardo - CESQA (Quality and Environmental Research Centre), Department of Industrial Engineering, University of Padova, 35131 Padova, Italy; 이이.org/ 0000-0003-0245-6944; Email: alessandro.manzardo@ unipd.it

\section{Authors}

Alessandro Marson - CESQA (Quality and Environmental Research Centre), Department of Industrial Engineering, 
University of Padova, 35131 Padova, Italy; 이이.org/ 0000-0003-4295-2328

Massimiliano Masiero - Department of Industrial Engineering, University of Padova, 35131 Padova, Italy

Michele Modesti - Department of Industrial Engineering, University of Padova, 35131 Padova, Italy

Antonio Scipioni - CESQA (Quality and Environmental Research Centre), Department of Industrial Engineering, University of Padova, 35131 Padova, Italy

Complete contact information is available at:

https://pubs.acs.org/10.1021/acsomega.0c05844

\section{Notes}

The authors declare no competing financial interest.

\section{ACKNOWLEDGMENTS}

The authors acknowledge the Department of Industrial Engineering of the University of Padova for providing the laboratory infrastructure and the resources to perform the research.

\section{REFERENCES}

(1) Nielsen, T. D.; Hasselbalch, J.; Holmberg, K.; Stripple, J. Politics and the Plastic Crisis: A Review throughout the Plastic Life Cycle. Wiley Interdiscip. Rev. Energy Environ. 2020, 9, No. e360.

(2) Boucher, J.; Dubois, C.; Kounina, A.; Puydarrieux, P. Review of Plastic Footprint Methodologies. 2019. https://doi.org/10.2305/ IUCN.CH.2019.10.en.

(3) PlasticsEurope. Plastics-The Facts 2019; 2019.

(4) European Union. Report: Attitudes of European Citizens towards the Environment; 2020.

(5) European Commission. Closing the Loop-An EU Action Plan for the Circular Economy, Brussels; 2015.

(6) UNEA. United Nations Environment Assembly of the United Nations Environment Programme 3/7. Marine Litter and Microplastics; 2017.

(7) WHO. Microplastics in Drinking Water; 2019.

(8) European Commission. A European Strategy for Plastics; 2018.

(9) IBF International Consulting. Support the Public Consultation on a New Circular Economy Action Plan; 2020.

(10) Sheldon, R. A. Metrics of Green Chemistry and Sustainability: Past, Present, and Future. ACS Sustainable Chem. Eng. 2018, 6, 3248 .

(11) Walker, S.; Rothman, R. Life Cycle Assessment of Bio-Based and Fossil-Based Plastic: A Review. J. Cleaner Prod. 2020, 261, No. 121158.

(12) Toniolo, S.; Mazzi, A.; Niero, M.; Zuliani, F.; Scipioni, A. Comparative LCA to Evaluate How Much Recycling Is Environmentally Favourable for Food Packaging. Resour. Conserv. Recycl. 2013, 77, 61-68.

(13) Broeren, M. L. M.; Molenveld, K.; van den Oever, M. J. A.; Patel, M. K.; Worrell, E.; Shen, L. Early-Stage Sustainability Assessment to Assist with Material Selection: A Case Study for Biobased Printer Panels. J. Cleaner Prod. 2016, 135, 30-41.

(14) Manzardo, A.; Marson, A.; Roso, M.; Boaretti, C.; Modesti, M.; Scipioni, A.; Lorenzetti, A. Life Cycle Assessment Framework To Support the Design of Biobased Rigid Polyurethane Foams. ACS Omega 2019, 4, 14114-14123.

(15) Bhunia, H. P.; Jana, R. N.; Basak, A.; Lenka, S.; Nando, G. B. Synthesis of Polyurethane from Cashew Nut Shell Liquid (CNSL), a Renewable Resource. J. Polym. Sci., Part A: Polym. Chem. 1998, 36, 391-400.

(16) Ionescu, M.; Radojčić, D.; Wan, X.; Shrestha, M. L.; Petrović, Z. S.; Upshaw, T. A. Highly Functional Polyols from Castor Oil for Rigid Polyurethanes. Eur. Polym. J. 2016, 84, 736-749.
(17) Fridrihsone, A.; Romagnoli, F.; Kirsanovs, V.; Cabulis, U. Life Cycle Assessment of Vegetable Oil Based Polyols for Polyurethane Production. J. Cleaner Prod. 2020, 266, No. 121403.

(18) Gama, N.; Ferreira, A.; Barros-Timmons, A. Polyurethane Foams: Past, Present, and Future. Materials 2018, 11, No. 1841.

(19) Simón, D.; Borreguero, A. M.; de Lucas, A.; Rodríguez, J. F. Recycling of Polyurethanes from Laboratory to Industry, a Journey towards the Sustainability. Waste Manage. 2018, 76, 147-171.

(20) You, K. K.; Durocher, D. T.; Kierkus, P. C.; Fishback, T. L. Chemical Recycling of Polyurethanes and Applications for the Recyclates. J. Cell. Plast. 1998, 34, 261-270.

(21) Gadhave, R. V.; Srivastava, S.; Mahanwar, P. A.; Gadekar, P. T. Recycling and Disposal Methods for Polyurethane Wastes: A Review. Open J. Polym. Chem. 2019, 09, 39-51.

(22) CEN. EN 15804:2012+A2:2019-Standards Publication Sustainability of Construction Works-Environmental Product Declarations-Core Rules for the Product Category of Construction Products; 2019.

(23) European Commission. Product Environmental Footprint Category Rules (PEFCRs) for Thermal Insulation; 2019.

(24) van der Harst, E.; Potting, J.; Kroeze, C. Comparison of Different Methods to Include Recycling in LCAs of Aluminium Cans and Disposable Polystyrene Cups. Waste Manage. 2016, 48, 565-583.

(25) ISO. Environmental Management-Life Cycle AssessmentPrinciples and Framework (ISO 14040:2006/Amd 1:2020); 2020.

(26) European Commission. Guidance for the Development of Product Environmental Footprint Category Rules (PEFCRs) v. 6.3; 2018; pp 1142.

(27) Allacker, K.; Mathieux, F.; Pennington, D.; Pant, R. The Search for an Appropriate End-of-Life Formula for the Purpose of the European Commission Environmental Footprint Initiative. Int. J. Life Cycle Assess. 2017, 22, 1441-1458.

(28) CEN. Thermal Insulation Products-Product Category Rules (PCR) for Factory Made and In-Situ Formed Products for Preparing Environmental Product Declarations; 2017.

(29) Wernet, G.; Bauer, C.; Steubing, B.; Reinhard, J.; Moreno-Ruiz, E.; Weidema, B. The Ecoinvent Database Version 3 (Part I): Overview and Methodology. Int. J. Life Cycle Assess. 2016, 21, 12181230.

(30) PRé Sustainability B.V. SimaPro; 2019.

(31) Frischknecht, R. Global Guidance for Life Cycle Impact Assessment Indicators Volume 1; UNEP, 2016; Vol. 1.

(32) van Zelm, R.; Huijbregts, M. A. J.; den Hollander, H. A.; van Jaarsveld, H. A.; Sauter, F. J.; Struijs, J.; van Wijnen, H. J.; van de Meent, D. European Characterization Factors for Human Health Damage of PM10 and Ozone in Life Cycle Impact Assessment. Atmos. Environ. 2008, 42, 441-453.

(33) de Bruijn, H.; van Duin, R.; Huijbregts, M. A. J. Eco-Efficiency in Industry and Science. In Handbook on Life Cycle Assessment; Guinee, J. B.; Gorree, M.; Heijungs, R.; Huppes, G.; Kleijn, R.; de Koning, A.; van Oers, L.; Wegener Sleeswijk, A.; Suh, S.; Udo de Haes, H. A., Eds.; Springer Netherlands: Dordrecht, 2002; Vol. 7.

(34) Goedkoop, M.; Oele, M.; Leijting, J.; Ponsioen, T.; Meijer, E. Introduction to LCA with SimaPro; 2016. 